\title{
Corneo-Scleral Rim Cultures: Donor Contamination A Case of Fungal Endophthalmitis Transmitted by K-Sol Stored Cornea
}

\author{
LYE P. FONG $\dagger$, D. GLADSTONE $\uparrow \dagger$, T.A. CASEY $\dagger$ \\ East Grinstead and Tunbridge Wells
}

\begin{abstract}
Summary
This retrospective study of 549 corneo-scleral rim cultures shows that gentamicin, used in MK and K-Sol medium storage at $4^{\circ} \mathrm{C}$, has decreased donor contamination from $43 \%$ in wholeglobe storage to $13 \%$, but failed to eliminate coagulase negative staphylococci $(37 \%)$, streptococci $(\mathbf{2 8} \%)$ and fungi $(\mathbf{2 8 \%})$. Donor-to-host transmitted staphylococcal and streptococcal endophthalmitis have been reported previously. We present the first documented case of donor-to-recipient transmitted fungal endophthalmitis following corneal transplantation using corneas stored in $\mathrm{MK}$ or $\mathrm{K}-\mathrm{Sol}$ solution at $4^{\circ} \mathrm{C}$; Candida albicans was isolated. Recommendations are made to assess critically the true incidence of donor fungal contamination and the necessity of adding anti-mycotic agents to preservation medium for $4^{\circ} \mathrm{C}$ storage. In the absence of ideal antimicrobial cover for corneal preservation solutions, stringent prophylactic measures to reduce contamination and continued monitoring of corneo-scleral rim cultures are warranted, if the poor visual consequences of donor-to-host transmitted endophthalmitis are to be avoided.
\end{abstract}

The conversion of corneal storage from the whole eye moist chamber technique to McCarey-Kaufman (MK) and K-Sol tissue culture media has coincided with increased interest in the risk of postoperative endophthalmitis from contaminated donor tissue and storage medium. ${ }^{1,2}$ Gentamicin, which is used alone in MK and K-Sol medium, is known to be highly effective against staphylococcus and Pseudomonas (Ps.) aeruginosa but recent reports have questioned its efficacy against certain strains of streptococcus ${ }^{3-5}$ in this storage medium.

These recent reports, together with our experience of two donor-related postkeratoplasty intraocular infections, one of which was fungal in origin, initiated this retrospective review of 549 corneo-scleral donor rim cultures obtained from corneas stored in MK and K-Sol medium or as whole globes. The objectives of this study were to:

(i) identify the spectrum of microbial contamination of donor corneas

(ii) suggest recommendations for reducing donor corneal contamination in storage media at $4^{\circ} \mathrm{C}$.

\section{Materials and Methods}

Over an eight year period, between 1980 and February 1988, 549 penetrating keratoplasty cases performed at the Corneo-Plastic Unit, Queen Victoria Hospital, East Grinstead, were reviewed. Donor details were recorded, including the donor age, cause of death, time 
from death to enucleation and method and duration of storage. The charts of the recipients were searched for the preoperative corneal diagnosis, the presence of pre-existing ocular infections, possible ocular or systemic factors predisposing to infections and the postoperative course for evidence of ocular infection. The minimum review period was two months but most patients' charts yielded information for extended periods of time, often over a course of years, either until the present date or referral to the local ophthalmologist.

The standardised procedures for corneal preparation were as follows: whole eyes were enucleated, within 12 hours of death, in an aseptic manner by someone medically qualified, using sterile instruments and drapes. The globes were supported in sterile containers, irrigated with $5 \mathrm{ml}$ of Neosporin ophthalmic solution (polymixin B-neomycingramicidin, Burroughs Wellcome Co.) and packed in ice for transport to the Eye Bank, within one to two hours. Further preparation was as follows: according to the method of corneal storage:

(i) Whole globes were stored at $4^{\circ} \mathrm{C}$ for up to 24 hours and then immersed in soframycin solution for 30 minutes prior to transplantation

(ii) $M K$ and $K$-Sol corneas were immediately prepared by dissecting the corneo-scleral discs with sterile instruments for immersion in storage medium at $4^{\circ} \mathrm{C}$.

Most MK and K-Sol corneas were used within twelve to forty-eight hours of death and the longest period of storage was four days. The MK and K-Sol stored corneas were then warmed to room temperature for half to one hour before transplantation.

No routine topical antibiotics were used preoperatively. At the end of surgery, subconjunctival injections of either gentamycin or tobramycin $(20 \mathrm{mg})$ and dexamethasone $(4 \mathrm{mg})$ were given and Oc. chloramphenicol $1 \%$ was instilled. G. chloramphenicol $1 \%$ and $\mathrm{G}$. dexamethasone $0.1 \%$ were used routinely, on a q.i.d. dosage, for one month postoperatively, after which the antibiotic was discontinued and steroid drops tapered off.

After excision of the donor disc for transplantation, all residual corneo-sceral rims were swabbed and plated on to the culture media: blood, chocolate and modified Sabouraud's agar at $37^{\circ} \mathrm{C}$; Sabouraud's agar was also incubated at $25^{\circ} \mathrm{C} .{ }^{6}$ Culture plates for bacterial isolates were held for at least three days and fungal cultures for a minimum of one week, before any negative results were confirmed. Organisms isolated were identified by standard diagnostic criteria and sensitivities were perfomed for the assumed pathogenic strains of bacteria using antibiotic disc criteria. ${ }^{6}$

\section{Results}

Of the 549 cases reviewed (Table I), 302 corneas were preserved in storage media, 251 in $\mathrm{MK}$ and 51 in $\mathrm{K}$-Sol solutions; $14 \%$ were culture positive and all, except one, were single isolates. Of 247 whole-globe stored corneas, $107(43 \%)$ were culture positive and 11 were polymicrobial. Analysis of the microbial spectrum showed that there were similar ratios of major pathogens in both the whole-globe and medium stored corneas. Gram-positive organisms accounted for approximately $70 \%$ of cases and of these, two-thirds were staphylococci, most being coagulase-negative staphylococci $(C N S)$ and one-third comprised streptococci. Onequarter of the cultures involved fungi.

During the study period, two patients developed post-keratoplasty endopthalmitis secondary to donor corneal contamination and their details are presented in Table II. Patient 1, who had a complex past ocular history and disorganised anterior segment from multiple surgical procedures, developed a Candida albicans keratitis and endophthalmitis; the same organism had been recovered from the K-Sol stored corneo-sceral rim three weeks previously. On the same operative list, the paired donor cornea, which was also stored in K-Sol solution and cultured Candida albicans, was used for a patient with pseudophakic keratopathy; his postoperative course was uneventful.

Ps. aeruginosa was isolated from the corneo-scleral rim of a whole globe in patient 2 , who had not received routine subconjunctival antibiotics at the time of surgery 
Table I. Culture results of corneo-scleral rims.

\begin{tabular}{lcc}
\hline & Corneal storage & \\
& MK and K-Sol & Whole globe \\
\hline Total number of cases & 302 & 247 \\
Number with culture positive rims & 43 & 106 \\
Polymicrobial cultures & 1 & 11 \\
Percentage of cases with positive cultures & 14 & 43 \\
Gram positive bacteria & & \\
Coagulase negative staphylococcus & $16(37 \%)$ & $36(31 \%)$ \\
Staphylococcus aureus & $1(2 \%)$ & $8(7 \%)$ \\
Viridans streptococcus & $5(12 \%)$ & $6(6 \%)$ \\
Streptococcus pneumoniae & $5(12 \%)$ & $1(1 \%)$ \\
Beta-haemolytic streptococcus & 0 & $6(5 \%)$ \\
Streptococcus faecalis & $2(5 \%)$ & $8(7 \%)$ \\
Unidentified streptococcus & 0 & $10(9 \%)$ \\
\hline Total number streptococcus & $12(28 \%)$ & $32(27 \%)$ \\
\hline Corynebacteria spp & $1(2 \%)$ & $7(6 \%)$ \\
Clostridia spp & 0 & $1(1 \%)$ \\
Gram negative bacteria & & $6(6 \%)$ \\
Pseudomonas aeruginosa & $2(5 \%)$ & $2(2 \%)$ \\
Proteus spp & 0 & $6(6 \%)$ \\
Escherichia spp & 0 & $33(31 \%)$ \\
Fungi (total number) & $12(28 \%)$ & $26(22 \%)$ \\
Candida albicans & $7(16 \%)$ & $3(3 \%)$ \\
Aspergillus spp & $2(5 \%)$ & 0 \\
Cladosporium spp & $2(5 \%)$ & $(3 \%)$ \\
Penicillium spp & $1(2 \%)$ & \\
\hline & & \\
\hline Denomina & & \\
\hline
\end{tabular}

*Denominator $=$ cases with positive cultures.

because of gentamicin hypersensitivity. Another two patients (unpublished data), whose MK-stored donor rims cultured $C N S$, developed hypopyons post-operatively but microbiological investigations were not instigated as the infection/inflammation resolved rapidly on intensive topical antibiotics and steroids.

The median age of the donors was 48 years (range four days to 82 years). The distribution of causes of death was uniform throughout both study groups and so combined figures are presented: acute myocardial infarction $34 \%$ carcinoma $30 \%$, road traffic accident $20 \%$ and cerebrovascular accident $18 \%$. Of eight donors with systemic infection, no organisms were isolated from two whole-globe specimens; three MK-stored corneas were culture negative; another three MK-preserved corneas, which cultured
Candida albicans, Aspergillus spp and CNS, were unrelated to the donor infective process; and none of the recipients developed post-operative infections.

\section{Discussion}

Although the use of high dose gentamicin $(100 \mathrm{microgm} / \mathrm{ml})$ in storage media has substantially reduced the donor contamination rate of whole-globe stored corneas $(43 \%)$ to $14 \%$, the residual donor contamination still poses a significant risk for donor-to-host transmitted infections. Leveille et $\mathrm{al}^{7}$ had shown a 22-fold increased incidence of endophthalmitis in patients with positive donor rim cultures when compared to those with negative results. Although the incidence for such donor-related infections is low, $0.4 \%$ for whole-globe stored corneas and $0.3 \%$ of MK and K-Sol storage in our series, the 
extremely poor visual outcome $\mathrm{e}^{4}$ justifies every precautionary measure to reduce and, ideally, eliminate all donor contaminants.

If aerobic cultures alone are considered, the $14 \%$ incidence of positive cultures in our series is comparable to $17 \%$ and $20 \%$ recorded by Mathers, ${ }^{5}$ Mascerella ${ }^{8}$ and their co-authors (Table III). They also found that $C N S$ and streptococci were the predominant potential pathogens in corneo-sceral cultures following $\mathrm{MK}$ storage. Although no infections were reported from their smaller

Table II. Summary of cases with post-keratoplasty endophthalmitis and culture-positive donor rim corneas

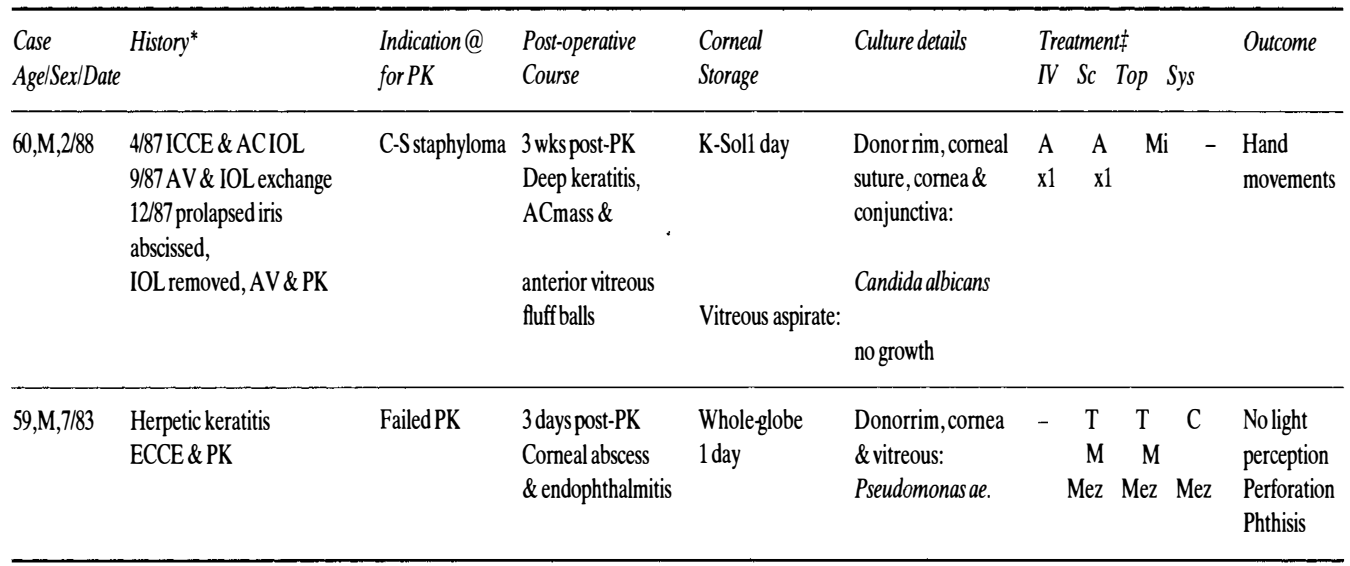

${ }^{*} \mathrm{ICCE}=$ Intracapsular cataract extraction, $\mathrm{AC}=$ Anterior chamber, $\mathrm{IOL}=$ Intraocular lens, $\mathrm{AV}=$ Anterior vitrectomy, ECCE $=$ Extracapsular cataract extraction, $\mathrm{PK}=$ Penetrating keratoplasty @ C-S = Corneo-scleral

$\ddagger \mathrm{A}=$ Amphotericin $\mathrm{B}$, IV 10 microgm, SC $0.3 \mathrm{mg} ; \mathrm{Mi}=$ Miconazole; $\mathrm{T}=$ Tobramycin; $\mathrm{C}=$ Cephalexin; $\mathbf{M}=$ Methicillin; $\mathrm{Mez}=$ Mezlocillin; IV = Intravenous; $\mathrm{SC}=$ Subconjunctival; Top = Topical; Sys $=$ Systemic.

Table III. Corneo-scleral rim cultures of corneas stored in $M K$ and $K$-sol medium

\begin{tabular}{llll}
\hline & Mascarella & Mathers & Fong \\
& 1979 & 1987 & 1988 \\
& $N=200$ & $N=291$ & $N=302$ \\
\hline Percentage of positive cultures & 12 & 39 & 14 \\
Propionibacterium spp & $36 \% *$ & $32 \%$ & ND@ \\
Coagulase-negative staphylococcus & $14 \%$ & $30 \%$ & $37 \%$ \\
Staphylococcus aureus & $7 \%$ & $3 \%$ & $2 \%$ \\
Streptococci & $11 \%$ & $9 \%$ & $28 \%$ \\
Corynebacteria spp & $14 \%$ & $8 \%$ & $2 \%$ \\
Pseudomonas aeruginosa & 0 & 0 & $5 \%$ \\
Proteus & $4 \%$ & $1 \%$ & 0 \\
Candida albicans & $4 \%$ & $1 \%$ & $16 \%$ \\
Aspergillus spp & 0 & 0 & $5 \%$ \\
Cladosporium spp & 0 & 0 & $5 \%$ \\
Fusarium solani & $4 \%$ & 0 & 0 \\
Torulopsis glabrata & $4 \%$ & 0 & 0 \\
Penicillium spp & 0 & 0 & $2 \%$ \\
\hline
\end{tabular}

* Denominator $=$ cases with positive cultures

@ ND = Not done 
series of cases, Leveille et $\mathrm{al}^{7}$ found a $0.2 \%$ incidence of donor-related post-operative endophthalmitis in 1,876 penetrating keratoplasties with MK stored corneas.

Review of our data has highlighted the need for a re-appraisal of our current eye bank techniques. Donors need to be preselected to exclude potentially contaminated material from septic donors. ${ }^{9,10}$ Further modifications of corneal preparation techniques to reduce the donor contamination rate include: mechanical irrigation with saline solution, chemical preparation of the external ocular surface with povidine-iodine, immersion rather than irrigation with antibiotics, the use of a laminar flowhood and a standardised period of warming of corneas and storage media from $4^{\circ} \mathrm{C} .{ }^{11-13}$

Gentamicin is effective against a wide range of organisms but, as our study shows, $C N S$, streptococci and fungi are resistant to this aminoglycoside, which is currently used for both MK and K-Sol media. Streptococci are particularly resistant, even with extended periods of warming-up time. ${ }^{4}$ Clinical reports by Matoba and Baer and their co-workers ${ }^{3,4}$ have emphasised the emergence of streptococci as major aetiological agents in the transmission of intra-ocular infections through contaminated MK stored tissue. Of the 15 cases where bacteria were recovered from either corneo-scleral rims or MK medium, one-third were due to streptococci. ${ }^{1,3,4}$

$P s$. aeruginosa is an infrequent external ocular contaminant. ${ }^{14}$ Two of our MK and KSol stored corneas cultured Ps. aeruginosa, albeit with no transmission of infection. One case of post-keratoplasty endophthalmitis secondary to MK-stored donor cornea contaminated with Ps. aeruginosa was reported by Leveille et al. ${ }^{7}$ It cannot be determined if these infrequent contaminations were due to particularly virulent strains, large inoculae, in vivo resistance to gentamicin or inadequate antimicrobial activity because of insufficient warming of the MK medium. ${ }^{4}$ Although the organism was sensitive to gentamicin in all three instances, the emergence of gentamicin-resistant strains remain an area of concern. ${ }^{15}$

A number of authors ${ }^{3-5}$ have suggested the addition of a second antibiotic, such as a cephalosporin or penicillin, to eliminate a greater range of organisms, but others have cautioned against the ineffectiveness of these antibiotics at $4^{\circ} \mathrm{C} . .^{4,16}$ Antibiotic activity is dependent on the rate of metabolism of bacteria, and therefore, on temperature. With "warming-up" from $4^{\circ} \mathrm{C}$, those antimicrobials affecting more "essential" metabolism, e.g. aminoglycosides on ribosomes and DNA synthesis, would be expected to be effective sooner than cell-wall agents, such as penicillins and cephalosporins. The newer fluoroquinolene antibiotics, which affect DNA gyrase activity in a broader spectrum of bacteria, are potentially useful $^{17}$ but more work is required to ascertain their efficacy and safety.

The microbiological data in this study may be improved by the following technical modifications:

(i) anaerobic cultures

(ii) elimination of pre-soaked antibiotics

(iii) cultures in brain heart infusion broth medium

(iv) maintaining fungal cultures for one month.

The high incidence of fungal recovery in our study $(28 \%)$ differs from the $0.5 \%$ incidence reported by Badenoch and coauthors $^{(18)}$ and $11 \%$ in Mascarella and Cavanagh's series. ${ }^{7}$ One explanation for the discrepancies may be short culture periods. Although most fungi grew within four days, O'Day and his co-workers ${ }^{(19)}$ showed that one-quarter were positive two to three weeks after inoculation and they recommended that both liquid and solid media be kept for four weeks before discarding. They also recommended the use of multiple media suitable for fungal growth, for which brainheart infusion medium was the most suitable.

The true incidence of fungal contamination, under effective eye bank decontamination procedures and laboratory conditions favourable to fungal growth, is unknown. Identification of this contamination risk, following $4^{\circ} \mathrm{C} \mathrm{MK}$ and $\mathrm{K}$-Sol storage, will help determine the necessity of supplementing anti-mycotic agents in storage media. ${ }^{20,21}$ Saggau and his co-authors ${ }^{22}$ observed a natural attrition of fungal organisms at $4^{\circ} \mathrm{C}$. 
Although the donor-to-host transmission of fungal infections from whole-globe and organ cultured corneas is well documented, ${ }^{8,21}$ our patient with Candida albicans is the first reported case of fungal infection transmitted through corneas stored in culture medium at $4^{\circ} \mathrm{C}$. The host immune defence system in our patient was likely to have been compromised, both from multiple surgical insults to the eye and longterm administration of topical antibiotics and steroids. It is interesting to note that no complications developed with the paired donor cornea which was grafted into a relatively healthy eye.

Identification of a culture-positive donor may aid in therapeutic guidelines for postkeratoplasty endophthalmitis, but microbiological confirmation of infection is still required. In the majority of our patients with positive donor rim cultures, the routine postoperative management was not altered, except for a few select patients, whose antibiotics were changed according to sensitivity data, depending on the inclination of the individual surgeon. Nevertheless, routine donor corneo-scleral cultures are required for monitoring quality control and the selection of antibiotics in culture media and perioperative care.

This study has confirmed that coagulase negative staphylococci $(37 \%)$, streptococci $(28 \%)$ and fungi $(28 \%)$ are resistant to gentimicin prophylaxis in $\mathrm{MK}$ and $\mathrm{K}$-Sol corneal storage at $4^{\circ} \mathrm{C}$. Our report of the first case of fungal infection transmitted by the donor cornea after MK or K-Sol sorage suggests that the true incidence of fungal contamination needs to be established to determine the necessity of adding antimycotic agents to the storage media for $4^{\circ} \mathrm{C}$. Despite the low incidence of donor-related post-keratoplasty infections, it is imperative to minimise the possibility of such devastating infections by relying on stringent decontamination procedures in the eye bank, continual monitoring of corneo-scleral rim cultures and the development of new antimicrobials either as a replacement or supplement to gentimicin.

\section{References}

${ }^{1}$ Girard LJ: Bacterial endophthalmitis following the use of contaminated preserved corneal tissue. Cornea 1982; 1: 255-7.

${ }^{2}$ LeFrancois M, Baum JL: Flavobacterium endophthalmitis following keratoplasty. Arch Ophthalmol 1976; 94: 1907-9.

${ }^{3}$ Matoba A, Moore MB, Merten JL, McCulley JP: Donor-to-host transmission of streptococcal infection by corneas stored in McCarey-Kaufman medium. Cornea 1984; 3: 105-8.

${ }^{4}$ Baer JC, Nirankari VS, Glaros DS: Streptococcal endophthalmitis from contaminated donor corneas after keratoplasty. Clinical and laboratory investigations. Arch Ophthalmol 1988; 106: 517-20.

${ }^{5}$ Mathers WD, Lemp MA. Corneal rim cultures. Cornea 1987; 6(3): 231-3.

6 Jones DB, Liesegang TJ, Robinson NM: Laboratory diagnosis of ocular infections. American Academy of Ophthalmology Manuals Program 1981.

${ }^{7}$ Leveille AS, McMullan D, Cavanagh HD: Endophthalmitis following penetrating keratoplasty. Ophthalmol 1983; 90: 38-9.

${ }^{8}$ Mascarella K, Cavanagh HD: Penetrating keratoplasty using McCarey-Kaufman preserved corneal tissue. South Med J 1979; 72: 1268-71.

${ }^{9}$ Gandhi SS, Lamberts DW, Perry HD: Donor to host transmission of disease via corneal transplantation. Surv Ophthalmol 1981; 25: 30611.

${ }^{10}$ Olson RJ, McMain, Slappey TE: Donor eye contamination. Ann Ophthalmol 1979; 11: 1875-8.

11 Pardos GJ, Gallagher MA: Microbial contamination of donor eyes. A retrospective study. Arch Ophthalmol 1982; 100: 1611-13.

12 Keates RH, Mishler KE, Riedinger D: Bacterial contamination of donor eyes. Am J Ophthalmol 1977; 84: 617-9.

13 Apt L, Isenberg S, Yoshimori R, Paez JH: Chemical preparation of the eye in ophthalmic surgery. III. Effect of povidone-iodine on the conjunctiva. Arch Ophthalmol 1984: 102: 728-9.

${ }^{14}$ Polack FN, Locatcher-Khorazo D, Gutierrez E: Bacteriologic study of "donor" eyes. Arch Ophthalmol 1967; 78: 219-25.

15 Poole TG, Insler MS: Contamination of donor cornea by gentamicin-resistant organisms. Am J Ophthalmol 1984; 97: 560-4.

${ }^{16}$ Baum J, Barza M, Kane A: Efficacy of penicillin $\mathrm{G}$, cefazolin, and gentamicin in $\mathrm{M}-\mathrm{K}$ medium at $4^{\circ} \mathrm{C}$. Arch Ophthalmol 1978; 96: 1262-4. 
${ }^{17}$ Viti A, Seetharama S, Snyder I, Schwab IR: Bacterial growth and the use of ciprofloxacin in MK media. Invest Ophthalmol Vis Sci Supp 1988; 29: 444.

${ }^{18}$ Badenoch PR, Alfrich SJ, Wedding TR, Coster DJ: Effectiveness of a decontamination method for donor corneas. Br J Ophthalmol 1988; 72: 225-7.

${ }^{19}$ O'Day DM, Akrabawi PL, Head WS, Ratner HB: Laboratory techniques in human and experimental fungal infections. $A m J$ Ophthalmol 1979; 87: 688-93.
${ }^{20}$ Kowalski RP, Sundar Raj CV, Stuart JC, Dunn DS: Antifungal synergism: A proposed dosage for corneal storage medium. Arch Ophthalmol 1985; 103: 250-6.

${ }^{21}$ Nelson JD, Mindrup EA, Chung CK, Lindstrom RL, Doughman DJ: Fungal contamination in organ culture. Arch Ophthalmol 1983; 101: 280-3.

${ }^{22}$ Saggau DD, Bourne WM, Sinkeldam IR, Roberts GD: Replication of fungi in K-Sol corneal preservation medium at $4^{\circ} \mathrm{C}$. Arch Ophthalmol 1986; 104: 1362-3. 